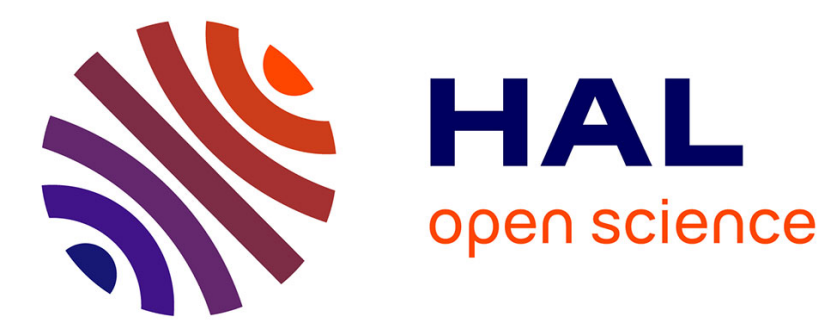

\title{
Sliding Mode Control Design for MIMO Systems: Implicit Lyapunov Function Approach
}

\author{
Andrey Polyakov, Denis Efimov, Wilfrid Perruquetti
}

\section{To cite this version:}

Andrey Polyakov, Denis Efimov, Wilfrid Perruquetti. Sliding Mode Control Design for MIMO Systems: Implicit Lyapunov Function Approach. European Control Conference, IEEE, IFAC, Jun 2014, Strasbourg, France. hal-01003984

\section{HAL Id: hal-01003984 \\ https://hal.inria.fr/hal-01003984}

Submitted on 11 Jun 2014

HAL is a multi-disciplinary open access archive for the deposit and dissemination of scientific research documents, whether they are published or not. The documents may come from teaching and research institutions in France or abroad, or from public or private research centers.
L'archive ouverte pluridisciplinaire HAL, est destinée au dépôt et à la diffusion de documents scientifiques de niveau recherche, publiés ou non, émanant des établissements d'enseignement et de recherche français ou étrangers, des laboratoires publics ou privés. 


\title{
Sliding Mode Control Design for MIMO Systems: Implicit Lyapunov Function Approach
}

\author{
Andrey Polyakov, Denis Efimov, Wilfrid Perruquetti
}

\begin{abstract}
A new approach to robust control design is considered. It is based on Implicit Lyapunov Function method. An algorithm of robust finite-time stabilization for a quasilinear multi-input disturbed system is developed. A new highorder sliding mode control algorithm is deduced. Procedures for tuning of control parameters are presented. They are based on Linear Matrix Inequalities (LMI). Aspects of practical implementation of developed algorithms are discussed. A scheme for chattering reduction is proposed. Theoretical results are supported by numerical simulations.
\end{abstract}

\section{INTRODUCTION}

Robustness issues are important for control systems applications. Sliding mode (SM) method is one of the classical approaches to robust control design [1], [2], [3]. It has a lot of extensions and specifications. The most famous recent achievement of sliding mode control theory is high order sliding mode (HOSM) control concept [4], [5]. HOSM algorithms provide some advantages with respect to classical SM schemes. For example, it may reduce chattering effects ("unmodeled" destructive oscillations of a closed-loop system). The theoretical background of HOSM control systems was developed since 1990s [6]. However, an application of existing HOSM control algorithms is complicated, since the only few constructive algorithms for tuning their parameters exist. Most of them are restricted to the second order case (see, for example, [5], [7]).

Finite-time stability property [8], [9], [10] is closely related to HOSM control analysis and design [11], [5], [12]. Frequently, a control law, which stabilizes a chain of integrators in finite-time and rejects bounded matched disturbances, belongs to a class of HOSM algorithms [4], [13]. A scheme for finite-time stabilization of a chain of integrators is also presented in [14]. However, it also does not have a constructive algorithms for parameters tuning.

Homogeneity property is very useful for HOSM control analysis [4], [11], [15]. Particulary, if an asymptotically

\footnotetext{
*This work was supported by EU Interreg IV A 2 Mers Seas Zeeën Cross-border Cooperation Programme under SYSIASS project 06-020.

Andrey Polyakov, Denis Efimov, Wilfrid Perruquetti are with Non-A INRIA - LNE, Parc Scientifique de la Haute Borne 40, avenue Halley Bat.A, Park Plaza 59650 Villeneuve d'Ascq(e-mail: andrey.polyakov@inria.fr, denis.efimov@inria.fr,wilfrid.perruetti@inria.fr). They are also with LAGIS UMR CNRS 8219, Ecole Centrale de Lille, BP 48, Cité Scientifique, 59651 Villeneuve-d'Ascq, France.

Denis Efimov is also with Department of Control Systems and Informatics, National Research University ITMO, 49 Kronverkskiy av., 197101 Saint Petersburg, Russia. This work was partially supported in part by the Government of Russian Federation (Grant 074-U01) and the Ministry of Education and Science of Russian Federation (Project 14.Z50.31.0031).

This work was partially supported by ANR Grant CHASLIM (ANR-11BS03-0007)
}

stable system is homogeneous of negative degree, then it is finite-time stable. However, even if a structure of homogenous HOSM control is defined, then anyway a design procedure has to be developed for a selection of control parameters, which guarantees at least asymptotic stability of a closed-loop system. An adjustment of convergence time is also very important in practice.

Lyapunov function method is the main approach to stability analysis and nonlinear control design. Lyapunov functions for existing second order SM algorithms can be found, for example, in [7], [16], [17]. Lyapunov analysis for HOSM algorithms of arbitrary order is still not well developed.

The present paper uses Implicit Lyapunov Function (ILF) method [18], [19], [20] for HOSM control design. The ILF structure is defined using weighted homogeneity approach [21], [22], [23] and some ideas from the papers [18], [24] and [25]. The obtained ILF has ellipsoidal level sets and provides LMI representation of stability conditions, which is important for simplicity of tuning of control parameters. The implicit Lyapunov analysis implies implicit definition of the control function, which requires special algorithms for practical ILF HOSM control implementations. Two of them are briefly discussed in the paper. This paper extends the finite-time ILF control method developed for a chain of integrators [20] to the multi-input linear systems. Moreover, the paper develops the new HOSM control algorithm, which guarantees finite-time stabilization of linear MIMO system and rejects bounded matched and "vanishing" unmatched disturbances of a certain type.

\section{Notation:}

- $\mathbb{R}$ is a set of real numbers; $\mathbb{R}_{+}=\{x \in \mathbb{R}: x>0\}$.

- $\|x\|$ denotes the euclidian norm of the vector $x \in \mathbb{R}^{n}$.

- range $(B)$ is a column space of a matrix $B \in \mathbb{R}^{n \times m}$.

- $\operatorname{diag}\left\{\lambda_{1}, \ldots, \lambda_{n}\right\}$ is a diagonal matrix with elements $\lambda_{i}$.

- The notation $P>0(<0, \geq 0, \leq 0)$ for $P \in \mathbb{R}^{n \times n}$ means that $P$ is symmetric and positive (negative) definite (semidefinite).

- A continuous function $\sigma: \mathbb{R}_{+} \rightarrow \mathbb{R}_{+}$belongs to the class $\mathcal{K}$ if it is monotone increasing and $\sigma(0)=0$.

\section{Problem Statement}

Consider the control system

$$
\dot{x}(t)=A x(t)+B u(t)+d(t, x)
$$

where $x \in \mathbb{R}^{n}$ is the state vector, $u \in \mathbb{R}^{m}$ is the vector of control inputs, $A \in \mathbb{R}^{n \times n}$ is the system matrix, $B \in \mathbb{R}^{n \times m}$ is the matrix of control gains and the function $d: \mathbb{R} \times \mathbb{R}^{n} \rightarrow$ 
$\mathbb{R}^{n}$ describes the system uncertainties and disturbances (or nonlinearities of the system).

It is assumed that the matrices $A$ and $B$ are known, the pair $(A, B)$ is controllable and the whole state vector $x$ can be measured and used for feedback control design.

Assumption 1: We also assume that the disturbance function $d(t, x)$ admits a decomposition into a bounded matched part and a vanishing unmatched one, i.e.

$$
d(t, x)=d_{m}(t, x)+d_{u m}(t, x),
$$

where $d_{m}(t, x) \in \operatorname{range}(B)$ is bounded and $d_{u m}(t, x) \perp \operatorname{range}(B)$ is "vanishing" at the origin, i.e. $\left\|d_{u m}(t, x)\right\| \rightarrow 0$ if $\|x\| \rightarrow 0$.

More rigorous restrictions to $d(t, x)$ are be given below.

The control aim is to stabilize the origin of the system (1) in a finite time. In addition, control algorithm has to reject bounded matched and "vanishing" unmatched disturbances by means of HOSM realization.

\section{FINITE TIME STABILITY AND IMPLICIT LyAPUNOV FUNCTION METHOD}

Consider the system of the form

$$
\dot{x}=f(t, x), \quad x(0)=x_{0},
$$

where $x \in \mathbb{R}^{n}$ is the state vector, $f: \mathbb{R}_{+} \times \mathbb{R}^{n} \rightarrow \mathbb{R}^{n}$ is a nonlinear vector field. Remark that piecewise continuous right-hand sides of the system (3) are not excluded. In this case the solutions $x\left(t, x_{0}\right)$ of the system (3) are understood in the sense of Filippov [27].

According to Filippov definition [27] an absolutely continuous function $x\left(t, x_{0}\right)$ is called a solution to the Cauchy problem associated to (3) if $x(0)=x_{0}$ and it satisfies the following differential inclusion

$$
\dot{x} \in K[f](t, x)=\operatorname{co} \bigcap_{\delta>0} \bigcap_{\mu(N)=0} f(t, x+B(\delta) \backslash N),
$$

where $\operatorname{co}(M)$ defines the convex closure of the set $M$ and the equality $\mu(N)=0$ means that the set $N \subset \mathbb{R}^{n}$ has measure 0 .

Assume that the origin is an equilibrium point of the system (3), i.e. $0 \in K[f](t, 0)$ for all $t \in \mathbb{R}$.

Definition 2 ([9], [8]): The origin of system (3) is said to be globally finite-time stable (FTS) if:

1) Finite-time attractivity: there exists a function $T: \mathbb{R}^{n} \backslash\{0\} \rightarrow \mathbb{R}_{+}$, such that for all $x_{0} \in$ $\mathbb{R}^{n} \backslash\{0\}, x\left(t, x_{0}\right)$ is defined on $\left[0, T\left(x_{0}\right)\right)$ and $\lim _{t \rightarrow T\left(x_{0}\right)} x\left(t, x_{0}\right)=0$.

2) Lyapunov stability: there exists a function $\delta \in \mathcal{K}$ such that for all $x_{0} \in \mathbb{R}^{n},\left\|x\left(t, x_{0}\right)\right\| \leq \delta\left(\left\|x_{0}\right\|\right)$.

The function $T$ is called the settling-time function of the system (3).

The next theorem presents recent result on the ILF method [19], [20]. This method admits analysis of the stability of the system without presenting the Lyapunov function in the explicit form. The Lyapunov function can be introduced in the implicit form, for example, as a solution of some algebraic equation: $Q(V, x)=0$. To analyze the stability of a system we do not need to solve this equation. It is enough to study (in some way) the equation itself together with the right-hand side of the system.

Theorem 3: [20] If there exists a continuous function

$$
\begin{gathered}
Q: \mathbb{R}_{+} \times \mathbb{R}^{n} \rightarrow \mathbb{R} \\
(V, x) \mapsto Q(V, x)
\end{gathered}
$$

such that

C1) $Q(V, x)$ is continuously differentiable $\forall x \in \mathbb{R}^{n} \backslash\{0\}$ and $\forall V \in \mathbb{R}_{+}$;

C2) for any $x \in \mathbb{R}^{n} \backslash\{0\}$ there exist $V^{-}, V^{+} \in \mathbb{R}_{+}$:

$$
Q\left(V^{-}, x\right)<0<Q\left(V^{+}, x\right)
$$

$C 3)$ for $\Omega=\left\{(V, x) \in \mathbb{R}^{n+1}: Q(V, x)=0\right\}$ we have

$$
\lim _{\substack{x \rightarrow 0 \\(V, x) \in \Omega}} V=0^{+}, \lim _{\substack{V \rightarrow 0^{+} \\(V, x) \in \Omega}}\|x\|=0, \lim _{\substack{\|x\| \rightarrow \infty \\(V, x) \in \Omega}} V=+\infty ;
$$

C4) $\frac{\partial Q(V, x)}{\partial V}<0, \forall V \in \mathbb{R}_{+}$and $\forall x \in \mathbb{R}^{n} \backslash\{0\}$;

C5) $\forall t \in \mathbb{R}_{+}, \forall(V, x) \in \Omega$

$$
\sup _{\forall y \in K[f](t, x)} \frac{\partial Q(V, x)}{\partial x} y \leq c V^{1-\mu} \frac{\partial Q(V, x)}{\partial V}
$$

where $c>0$ and $0<\mu<1$ are some constants, then the origin of system (3) is globally finite time stable with the following settling time estimate $T\left(x_{0}\right) \leq \frac{V_{0}^{\mu}}{c \mu}$, where $V_{0} \in \mathbb{R}_{+}: Q\left(V_{0}, x_{0}\right)=0$.

\section{CONTRol Design USing Implicit Lyapunov FUNCTION METHOD}

\section{A. Block Decomposition}

Introduce notations: $\operatorname{rown}(W)$ is a number of rows of a matrix $W$; $\operatorname{null}(W)$ is a matrix with columns defining an orthonormal basis of a null space of a matrix $W$.

Let us initially decompose the original multi input system (1) to a block from [26]. The required coordinate transformation can be constructed using supporting matrices provided by the following algorithm [17].

\section{Algorithm 4:}

Initialization : $A_{0}=A, B_{0}=B, T_{0}=I_{n}, k=0$. Recursive Step: While $\operatorname{rank}\left(B_{k}\right)<\operatorname{rown}\left(A_{k}\right)$ do

$$
\begin{gathered}
A_{k+1}=B_{k}^{\perp} A_{k}\left(B_{k}^{\perp}\right)^{T}, \quad B_{k+1}=B_{k}^{\perp} A_{k} \tilde{B}_{k} \\
T_{k+1}=\left(\begin{array}{c}
B_{k}^{\perp} \\
\tilde{B}_{k}
\end{array}\right), k=k+1
\end{gathered}
$$

where $B_{k}^{\perp}:=\left(\operatorname{null}\left(B_{k}^{T}\right)\right)^{T}, \tilde{B}_{k}:=\left(\operatorname{null}\left(B_{k}^{\perp}\right)\right)^{T}$.

This algorithm can be easily realized in a computational software system such as MATLAB.

Lemma 5: [17] If the pair $(A, B)$ is controllable then

1) Algorithm 4 terminates after a finite number of steps $k \leq n-1$;

2) the matrices $T_{i} \in \mathbb{R}^{\text {rown }\left(B_{i}\right) \times \operatorname{rown}\left(B_{i}\right)}, i=1,2, \ldots, k$ are orthogonal; 
3) for the orthogonal matrix

$$
\begin{gathered}
G=\left(\begin{array}{cc}
T_{k} & 0 \\
0 & I_{w_{k}}
\end{array}\right)\left(\begin{array}{cc}
T_{k-1} & 0 \\
0 & I_{w_{k-1}}
\end{array}\right) \ldots\left(\begin{array}{cc}
T_{2} & 0 \\
0 & I_{w_{2}}
\end{array}\right) T_{1}, \\
\text { where } w_{i}:=n-\operatorname{rown}\left(T_{i}\right)
\end{gathered}
$$

we have

$$
\begin{gathered}
G A G^{T}=\left(\begin{array}{ccccc}
A_{11} & A_{12} & 0 & \ldots & 0 \\
\ldots & \ldots & \ldots & \ldots & \ldots \\
A_{k-11} & A_{k-12} & A_{k-13} & \ldots & A_{k-1 k} \\
A_{k 1} & A_{k 2} & A_{k 3} & \ldots & A_{k k}
\end{array}\right), \\
G B=\left(\begin{array}{llllll}
0 & 0 & \ldots & 0 & A_{k k+1}^{T}
\end{array}\right)^{T},
\end{gathered}
$$

where $A_{k k+1}=\tilde{B}_{0} B_{0}, A_{i j} \in \mathbb{R}^{n_{i} \times n_{j}}, n_{i}:=\operatorname{rank}\left(B_{k-i}\right)$, $i, j=1,2, \ldots, k$ and $\operatorname{rank}\left(A_{i+1}\right)=n_{i}$.

Remark, if $B$ is a full rank matrix then $A_{k k+1}$ is square and nonsingular.

Since $\operatorname{rank}\left(A_{i i+1}\right)=\operatorname{rown}\left(A_{i i+1}\right)=n_{i}$ then $A_{i+1} A_{i i+1}^{T}$ is invertible and $A_{i i+1}^{+}=A_{i+1}^{T}\left(A_{i+1} A_{i i+1}^{T}\right)^{-1}$ is the right inverse matrix of $A_{i i+1}$.

Introduce the coordinate transformation [17] $s=\Phi y, s=$ $\left(s_{1}, \ldots, s_{k}\right)^{T}, s_{i} \in \mathbb{R}^{n_{i}}, y=\left(y_{1}, \ldots, y_{k}\right)^{T}, y_{i} \in \mathbb{R}^{n_{i}}$ by the recurrent formulas:

$$
\begin{gathered}
s_{i}=y_{i}+\varphi_{i}, i=1,2, \ldots, k, \varphi_{1}=0 \\
\varphi_{i+1}=A_{i i+1}^{+}\left(\sum_{j=1}^{i} A_{i j} y_{j}+\sum_{r=1}^{i} \frac{\partial \varphi_{i}}{\partial y_{r}} \sum_{j=1}^{r+1} A_{r j} y_{j}\right) .
\end{gathered}
$$

The presented coordinate transformation is linear and nonsingular. The inverse transformation $y=\Phi^{-1} s$ is defined as follows: $y_{i}=s_{i}+\psi_{i}, i=1,2, \ldots, k, \psi_{1}=0$, $\psi_{i+1}=A_{i i+1}^{+}\left(\sum_{k=1}^{i} \frac{\partial \psi_{i}}{\partial s_{k}} A_{i i+1} s_{k+1}-\sum_{j=1}^{i} A_{i j}\left(s_{j}+\psi_{j}\right)\right)$.

For example, if $k=3$ the matrix $\Phi$ has the form

$\Phi=\left(\begin{array}{ccc}I_{n_{1}} & 0 & 0 \\ A_{12}^{+} A_{11} & I_{n_{2}} & 0 \\ A_{23}^{+}\left(A_{21}+A_{12}^{+} A_{11}^{2}\right) & A_{23}^{+}\left(A_{22}+A_{12}^{+} A_{11} A_{12}\right) & I_{n_{3}}\end{array}\right)$

In the general case, the transformation $\Phi$ can be calculated numerically in MATLAB.

Applying the transformation $s=\Phi G x$ to the system (1) we obtain

$$
\dot{s}=\left(\begin{array}{ccccc}
0 & A_{12} & 0 & \ldots & 0 \\
\ldots & \ldots & \ldots & \ldots & \ldots \\
0 & 0 & \ldots & 0 & A_{k-1 k} \\
\tilde{A}_{k 1} & \tilde{A}_{k 2} & \tilde{A}_{k 3} & \ldots & \tilde{A}_{k k}
\end{array}\right) s+B^{\prime} u+\tilde{d},
$$

where the block $\tilde{A}_{k i}$ has the same size as $A_{k i}, B^{\prime}=\Phi G B=$

$$
\begin{aligned}
\left(\begin{array}{lllll}
0 & 0 & \ldots & 0 & A_{k+1}^{T}
\end{array}\right)^{T} \text { and } \\
\\
\tilde{d}:=\tilde{d}(t, s)=\Phi G d\left(t, G^{T} \Phi^{-1} s\right) .
\end{aligned}
$$

Let us select the control law in the form

$$
u=A_{k k+1}^{+}\left(\tilde{u}-K_{l i n} s\right),
$$

where $K_{l i n}=\left(\begin{array}{lllll}\tilde{A}_{k 1} & \tilde{A}_{k 2} & \ldots & \tilde{A}_{k k-1} & \tilde{A}_{k k}\end{array}\right)$ and $\tilde{u} \in$ $\mathbb{R}^{n_{k}}$ is the nonlinear part of feedback, which has to be designed in order to guarantee finite-time stability of the origin of the system:

$$
\dot{s}=\tilde{A} s+\tilde{B} \tilde{u}+\tilde{d}(t, s),
$$

where $s=\Phi G x$ and

$$
\begin{aligned}
\tilde{A} & =\left(\begin{array}{ccccc}
0 & A_{12} & 0 & \ldots & 0 \\
\ldots & \ldots & \ldots & \ldots & \ldots \\
0 & 0 & 0 & \ldots & A_{k-1 k} \\
0 & 0 & 0 & \ldots & 0
\end{array}\right), \\
\tilde{B} & =\left(\begin{array}{cccccc}
0 & 0 & \ldots & 0 & I_{n_{k}}
\end{array}\right)^{T} \in \mathbb{R}^{n \times n_{k}} .
\end{aligned}
$$

Remark 6: Feedback linearizable nonlinear systems $\dot{x}=$ $f(x)+g(x) u+d(t, x)$ can also be transformed into the form (11) (see, for example, [28]).

\section{B. Finite-time stabilization in the disturbance-free case}

Introduce the function

$$
Q(V, s):=s^{T} D_{r}\left(V^{-1}\right) P D_{r}\left(V^{-1}\right) s-1,
$$

where $s=\left(s_{1}, \ldots, s_{k}\right)^{T}, s_{i} \in \mathbb{R}^{n_{i}}, V \in \mathbb{R}^{+}, D_{r}(\lambda)$ is the dilation matrix of the form

$$
\begin{aligned}
D_{r}(\lambda)= & \left(\begin{array}{cccc}
\lambda^{r_{1}} I_{n_{1}} & 0 & \ldots & 0 \\
0 & \lambda^{r_{2}} I_{n_{2}} & \ldots & 0 \\
\ldots & \ldots & \ldots & \ldots \\
0 & \ldots & 0 & \lambda^{r_{k}} I_{n_{k}}
\end{array}\right), \quad \lambda>0, \\
& r_{i}=1+(k-i) \mu, \quad i=1,2, . ., k,
\end{aligned}
$$

$0<\mu \leq 1$ and $P \in \mathbb{R}^{n \times n}$ is a symmetric positive definite matrix, i.e. $P=P^{T}>0$. Denote $H_{\mu}:=\operatorname{diag}\left\{-r_{i} I_{n_{i}}\right\}_{i=1}^{k}$.

Theorem 7: If $\tilde{d}(t, s) \equiv 0$ and the system of matrix inequalities:

$$
\left\{\begin{array}{c}
\tilde{A} X+X \tilde{A}^{T}+\tilde{B} Y+Y^{T} \tilde{B}^{T}+\alpha X \leq 0 \\
-\gamma X \leq X H_{\mu}+H_{\mu} X<0, \quad X>0
\end{array}\right.
$$

is feasible for some $\alpha, \gamma \in \mathbb{R}_{+}, \mu \in(0,1]$ and $X \in \mathbb{R}^{n \times n}$, $Y \in \mathbb{R}^{m \times n}$ then the control of the form (10) with

$$
\tilde{u}=\tilde{u}(V, s)=V^{1-\mu} K D_{r}\left(V^{-1}\right) s,
$$

where $K:=Y X^{-1}$,

$$
V \in \mathbb{R}_{+}: Q(V, s)=0
$$

and $Q(V, s)$ is defined by (13) with $P:=X^{-1}$, stabilizes the origin of the system (1) in finite time and the settling time function is bounded as follows

$$
T\left(x_{0}\right) \leq \frac{\gamma V_{0}^{\mu}}{\alpha \mu},
$$

where $V_{0} \in \mathbb{R}_{+}: Q\left(V_{0}, \Phi G x_{0}\right)=0$.

Remark 8: The practical implementation of the control (16) requires solving of the equation $Q(V, s)=0$ in order to obtain $V(s)$. In some cases (for example, $k=2, \mu=1$ ), the function $V$ can be found analytically. In other cases this equation can be solved numerically and on-line during digital implementation of a control law. Two different implementation approaches are discussed in Section V.

Remark 9: The system of matrix inequalities (15) is feasible at least for sufficiently small $\mu \in(0,1]$ and sufficiently large $\gamma \in \mathbb{R}_{+}$. It can be easily solved using LMI toolbox of MATLAB or, for example, SeDuMi solver. The parameters $\gamma$ and $\alpha$ are introduced in the LMI system (15) in order to tune the convergence time (see, formula (17)). 
Remark 10: For $\mu \in(0,1)$ the control of the form (16) is continuous function of the state $x$.

If $\mu=1$ then the control function $\tilde{u}$ is continuous outside the origin and bounded for all $x \in \mathbb{R}^{n}$. Indeed, since $s^{T} D_{r}\left(V^{-1}\right) P D_{r}\left(V^{-1}\right) s=1 \Rightarrow\left\|D_{r}\left(V^{-1}\right) s\right\|^{2} \leq \frac{1}{\lambda_{\min }(P)}$ then for $\mu=1$ we have $\tilde{u}^{2} \leq\|K\|^{2} \cdot\left\|D_{r}\left(V^{-1}\right) s\right\|^{2} \leq$ $\frac{\|K\|^{2}}{\lambda_{\min }(P)}$, where $\lambda_{\min }(P)$ is a minimum eigenvalues of $P$.

Hence, it is easy to see that for $\mu=1$ in order to restrict the control magnitude by $\|\tilde{u}\| \leq u_{0}$ the following matrix inequality

$$
\left(\begin{array}{cc}
X & Y^{T} \\
Y & u_{0}^{2} I_{m}
\end{array}\right) \geq 0
$$

can be added to (15).

The control law (16) will be called Implicit Lyapunov Function-based control (or shortly ILF control).

\section{Disturbance Rejection and High Order Sliding Mode Control}

The presence of disturbances requires robust modification of the presented control design scheme. Denote

$$
E_{i}=\left(\begin{array}{ccccc}
0_{n_{1}} & \ldots & 0_{n_{1} \times n_{i}} & \ldots & 0_{n_{1} \times n_{k}} \\
\ldots & \ldots & \ldots & \ldots & \ldots \\
0_{n_{i} \times n_{1}} & \ldots & I_{n_{i}} & \ldots & 0_{n_{i} \times n_{k}} \\
\ldots & \ldots & \ldots & \ldots & \ldots \\
0_{n_{k} \times n_{1}} & \ldots & 0_{n_{k} \times n_{i}} & \ldots & 0_{n_{k}}
\end{array}\right)
$$

Theorem 11: If $\mu \in(0,1]$ and

1) the disturbance function $\tilde{d}$ satisfies the inequalities

$$
\tilde{d}^{T} E_{i} \tilde{d} \leq \beta_{i}^{2}\left\{\begin{array}{lll}
\left(s^{T} s\right)^{1+(k-i-1) \mu} & \text { if } \quad & s^{T} s \leq 1, \\
\left(s^{T} s\right)^{\frac{1+(k-i-1) \mu}{1+(k-1) \mu}} & \text { if } \quad & s^{T} s>1,
\end{array}\right.
$$

for some $\beta_{i} \geq 0, i=1,2, \ldots, k$;

2) for some $\alpha, \gamma \in \mathbb{R}_{+}$the system of linear matrix inequalities

$$
\begin{gathered}
\tilde{A} X+X \tilde{A}^{T}+\tilde{B} Y+Y^{T} \tilde{B}^{T}+\alpha X+\sum_{i=1}^{k} \beta_{i} E_{i} \leq 0 \\
-\gamma X \leq X H_{\mu}+H_{\mu} X<0, \alpha>\sum_{i=1}^{k} \beta_{i}, I_{n} \geq X>0 \\
X \in \mathbb{R}^{n \times n}, \quad Y \in \mathbb{R}^{m \times n}
\end{gathered}
$$

is feasible;

3) the control $\tilde{u}=\tilde{u}(V, s)$ has the form (16) with $P:=$ $X^{-1}$ and $K=Y X^{-1}$;

then the closed-loop system (1), is globally finite time stable and the settling time function estimate has the form

$$
T\left(x_{0}\right) \leq \frac{\gamma V_{0}^{\mu}}{\left(\alpha-\sum_{i=1}^{k} \beta_{i}\right) \mu}
$$

where $V_{0} \in \mathbb{R}_{+}: Q\left(V_{0}, \Phi G x_{0}\right)=0$.

It is important to remark that Assumption 1 is necessary for fulfilling (20). Indeed, if unmatched disturbances are not "vanishing" for $x \rightarrow 0$, then condition (20) never holds for $i=1,2, \ldots, k-1$.

Remark 12: If $\mu=1$ then restriction to the matched part of disturbances become the form $\tilde{d}^{T} E_{k} \tilde{d} \leq \beta_{k}^{2}$, i.e. the ILF control algorithm rejects bounded matched disturbances.
If $\tilde{d}^{T} E_{i} \tilde{d}=0$ for $i=1,2, \ldots, k-1$ then the algorithm guarantees that $\dot{s}_{1}(t)=\ddot{s}_{1}(t)=\ldots=s_{1}^{(k-1)}(t)=0$ after a finite period of time. Therefore, ILF control for $\mu=1$ realizes a high order sliding mode (HOSM). Remark 10 underlines that HOSM ILF control is discontinuous only at the origin similarly to Quasi-Continuous HOSM control [4].

\section{Practical IMPlEMENTATiON OF CONTROL ALGORITHM}

\section{A. Sampled-Time Implementation}

Practical implementation of the control algorithm (16) needs knowing $V(s)$. For $n=2, m=1$ the function $V$ can be calculated analytically. However, even for the second order case this representation is very cumbersome.

The function $V$ can be approximated numerically on a grid, which is constructed in some operation domain (a neighborhood of the origin).

The ILF control can be implemented in digital control devices that allow us to calculate a value of $V$ at some point $s$ by means of resolving the equation $Q(V, s)=0$ numerically and on-line.

Denote $V_{i}:=V\left(t_{i}\right)$ and $s_{i}:=s\left(t_{i}\right)$. On the time interval $\left[t_{i}, t_{i+1}\right)$ the control has the form $\tilde{u}\left(V_{i}, s\right)$.

It can be easily shown that for any fixed $V_{i}>0$ the control $\tilde{u}\left(V_{i}, s\right)$ is a linear stabilizing feedback for the system (11) without disturbances. The simple scheme for selection of the switching parameter $V_{i}$ is presented by the following algorithm [20].

Algorithm 13:

$$
\begin{aligned}
& \text { INITIALIZATION: } V_{0}=1 ; a=V_{\min } ; \quad b=1 \text {; } \\
& \text { STEP : } \\
& \text { If } x_{i}^{T} D_{r}\left(b^{-1}\right) P D_{r}\left(b^{-1}\right) x_{i}>1 \text { then } \\
& a=b ; \quad b=2 b \text { ； } \\
& \text { elseif } x_{i}^{T} D_{r}\left(a^{-1}\right) P D_{r}\left(a^{-1}\right) x_{i}<1 \text { then } \\
& b=a ; \quad a=\max \left\{\frac{a}{2}, V_{\min }\right\} \text {; } \\
& \text { else } \\
& c=\frac{a+b}{2} ; \\
& \text { If } x_{i}^{T} D_{r}\left(c^{-1}\right) P D_{r}\left(c^{-1}\right) x_{i}<1 \text { then } b=c \text {; } \\
& \text { else } a=\max \left\{V_{\min }, c\right\} \text {; } \\
& \text { endif: } \\
& \text { endif; } \\
& V_{i}=b \text {; }
\end{aligned}
$$

If $x_{i} \in \mathbb{R}^{n}$ is some given vector and STEP of this algorithm is applied recurrently many times to the same $x_{i}$ then Algorithm 13 realizes: 1) a localization of the unique positive root of the equation $\left.Q\left(V, x_{i}\right)=0 ; 2\right)$ improvement of the obtained localization by means of the bisection method.

Such an application of Algorithm 13 allows us to calculate $V\left(x_{i}\right)$ with high precision but it requests a high computational capability of a control device. If the computational power is very restricted, then STEP of Algorithm 13 may be realized just once at each sampled time instant.

The parameter $V_{\min }$ defines lower possible value of $V$. This parameter cannot be selected arbitrary small due to finite numerical precision of digital devices. 


\section{B. Adaptive Implementation}

An adaptive scheme can also be presented for practical implementation of ILF control.

Consider the system (11) without disturbances. In this case we have the following dynamic equation for the ILF:

$$
\dot{V}=-\frac{\left.s^{T} D_{r}\left(V^{-1}\right)(\tilde{A}+\tilde{B} K)^{T} P+P(\tilde{A}+\tilde{B} K)\right) D_{r}\left(V^{-1}\right) s}{s^{T}\left(P H_{\mu}+H_{\mu} P\right) s} V^{1-\mu} .
$$

If this equation is calculated on-line with the initial condition $V(0)=V_{0}>0: s(0)^{T} D_{r}\left(V_{0}^{-1}\right) P D_{r}\left(V_{0}^{-1}\right) s(0)=1$ then the corresponding trajectory $s(t)$ of the system (8) with the control $\tilde{u}(V(t), s)$ converges to the origin in a finite time according Theorem 7 .

In order to provide some robustness to the presented scheme the dynamic equation (22) can be modified as follows

$$
\dot{V}=-H[-Q(V, s)] \frac{s^{T} D_{r}\left(V^{-1}\right) Q_{1} D_{r}\left(V^{-1}\right) s}{s^{T} Q_{2} s} V^{1-\mu},
$$

where $Q_{1}=(\tilde{A}+\tilde{B} K)^{T} P+P(\tilde{A}+\tilde{B} K), Q_{2}=P H_{\mu}+H_{\mu} P$ and $H$ is a Heaviside step function

$$
H[\rho]=\left\{\begin{array}{lll}
1 & \text { for } & \rho \geq 0 \\
0 & \text { for } & \rho<0 .
\end{array}\right.
$$

The modified dynamic equation (23) guarantees that the function $V$ will be decreasing only in the case $s^{T}(t) D_{r}\left(V^{-1}(t)\right) P D_{r}\left(V^{-1}(t)\right) s(t) \leq 1$, i.e. if $s$ belongs to the ellipsoidal level set of the ILF. Otherwise, $\dot{V}=0$ and the function $V$ is constant. Since $\tilde{u}(V, s)$ is a linear stabilizing feedback for any fixed $V>0$ then the adaptive scheme (23) of the ILF implementation will guarantee at least asymptotic stabilization of the system (11) without disturbances. For the chattering reduction the function $V$ can also be restricted from below by some minimal value $V_{\min }$.

A more detailed study of the ILF control implementation goes out of the scope of this paper. It is suggested as a topic for future research. The numerical simulations given below demonstrate an effectiveness of the presented simplest algorithms of ILF control implementation even for disturbed cases.

\section{EXAMPLE}

Consider the system (1) with parameters

$$
A=\left(\begin{array}{ccccc}
\frac{1}{2} & \frac{-1}{10} & -1 & \frac{1}{10} & 0 \\
0 & 1 & \frac{2}{5} & \frac{1}{2} & -1 \\
\frac{-7}{10} & 1 & \frac{-1}{2} & 1 & \frac{-1}{5} \\
1 & \frac{1}{2} & -1 & \frac{3}{10} & 1 \\
2 & \frac{-3}{10} & 1 & \frac{-1}{2} & -2
\end{array}\right), B=\left(\begin{array}{cc}
1 & 0 \\
0.1 & 0.5 \\
0 & 1 \\
0 & 0 \\
0.7 & -0.6
\end{array}\right) .
$$

The block decomposition procedure presented in Section IVA gives the following coordinate transformations

$$
G=\left(\begin{array}{ccccc}
-0.1763 & -0.7162 & 0.5706 & -0.0703 & 0.3541 \\
-0.0087 & -0.0544 & 0.0393 & 0.9975 & 0.0202 \\
-0.5120 & 0.5442 & 0.1201 & 0.0073 & 0.6537 \\
-0.0100 & -0.3962 & -0.7904 & 0 & 0.4672 \\
0.8406 & 0.1760 & 0.1838 & 0 & 0.4782
\end{array}\right),
$$

$$
\Phi=\left(\begin{array}{ccccc}
1.0000 & 0 & 0 & 0 & 0 \\
0.0082 & 1.0000 & 0 & 0 & 0 \\
-0.3596 & 0 & 1.0000 & 0 & 0 \\
0.1443 & 0.0261 & 0.5160 & 1 & 0 \\
-0.7476 & 0.1778 & -0.2217 & 0 & 1
\end{array}\right) .
$$

with the sizes of the blocks $n_{1}=1, n_{2}=2, n_{3}=2$. The control is defined by the formula (10) with

$$
A_{34}^{+}=\left(\begin{array}{cc}
-0.0100 & 0.8406 \\
-0.7904 & 0.1838
\end{array}\right)
$$

and the following gain matrix for linear part of the control:

$$
K_{\text {lin }}=\left(\begin{array}{ccccc}
1.3362 & -1.3029 & -1.4298 & -1.3028 & 1.1285 \\
-0.9830 & -0.0173 & -0.8415 & 0.3545 & 0.6028
\end{array}\right) \text {. }
$$

According to the formula (12) the matrix $\tilde{A}$ is formed of the following blocks $A_{12}=\left(\begin{array}{ll}0.0076 & -0.3355\end{array}\right)$, $A_{23}=\left(\begin{array}{cc}1.0676 & 1.2241 \\ -2.1299 & 0.2598\end{array}\right)$.

We assume that the disturbance function $\tilde{d}$ defined by (9) satisfies the condition (20) with $\beta_{1}=0.01, \beta_{2}=0.16, \beta_{3}=$ 0.42. The function

$$
d=\frac{1}{5}\left(\begin{array}{c}
\sin (t) \\
0.5 \cos \left(x_{2}\right)+0.1 \sin (t) \\
\cos \left(x_{2}\right) \\
x_{1} x_{3} /\left(3+\|x\|^{2}\right) \\
0.6 \cos \left(x_{2}\right)+0.7 \sin (t)
\end{array}\right)
$$

which is used for simulations, satisfies the conditions (20) with selected $\beta_{i}, i=1,2,3$. The nonlinear part of the control is defined by the formula (16) for $\mu=1$ (HOSM case). Its parameters are designed in MATLAB using LMI (21) under additional restriction $\|\tilde{u}\| \leq u_{0}:=2$ expressed by LMI (18) for $\alpha=0.5$ and $\gamma=7.5$ :

$$
\begin{aligned}
P & =\left(\begin{array}{ccccc}
15.9067 & -0.2465 & -8.2320 & 2.8292 & -0.9992 \\
-0.2465 & 2.4381 & 0.2688 & -0.0444 & 1.0175 \\
-8.2320 & 0.2688 & 5.7332 & -1.8384 & 0.6414 \\
2.8292 & -0.0444 & -1.8384 & 2.2735 & -0.1347 \\
-0.9992 & 1.0175 & 0.6414 & -0.1347 & 2.0535
\end{array}\right), \\
K & =\left(\begin{array}{ccccc}
-11.4765 & -1.0150 & 7.9793 & -4.5694 & 0.3002 \\
2.5416 & -2.9191 & -1.8223 & 0.3238 & -3.5726
\end{array}\right) .
\end{aligned}
$$

The simulation results for ILF HOSM control application are depicted on Fig. 1. The initial condition is selected as $x(0)=$ $(2,3,-2,0,-2.4)^{T}$. The Fig. 2 presents the control signals and the equivalent control $u^{e q}$ obtained from the equation $0=B u^{e q}+d(t, 0), u^{e q} \in \mathbb{R}^{2}$. The last system of algebraic equations with respect to $u^{e q}$ is consistent, since $d(t, 0) \in$ range $B$, i.e. all "nonvanishing" disturbances are matched.

Numerical calculations of the closed-loop system have been done using the Euler method with the step size $h=0.1$. Such step size is quite a large for sliding mode control application, since it may provoke a chattering phenomenon even during the simulations. However, the control is applied using Algorithm 13, which allows to reduce the chattering by means of tuning the parameter $V_{\text {min }}$. For simulations it was selected as follows $V_{\min }=0.3$. This scalar parameter has 


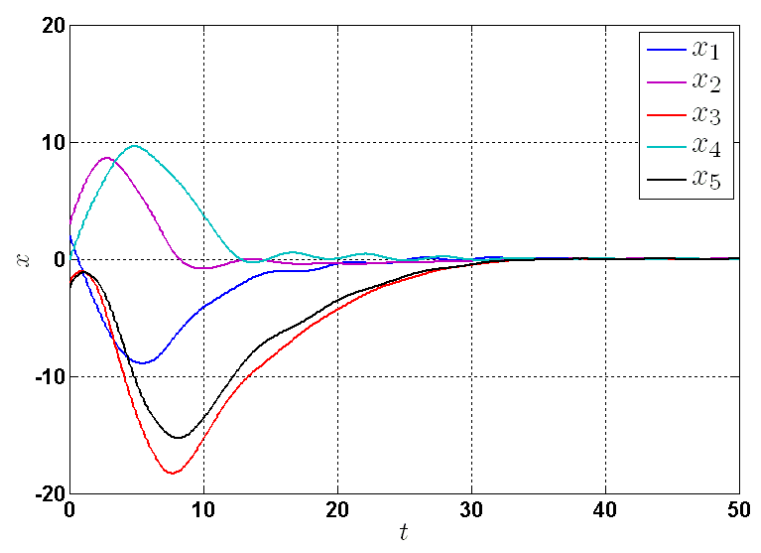

Fig. 1. Evolution of system states.

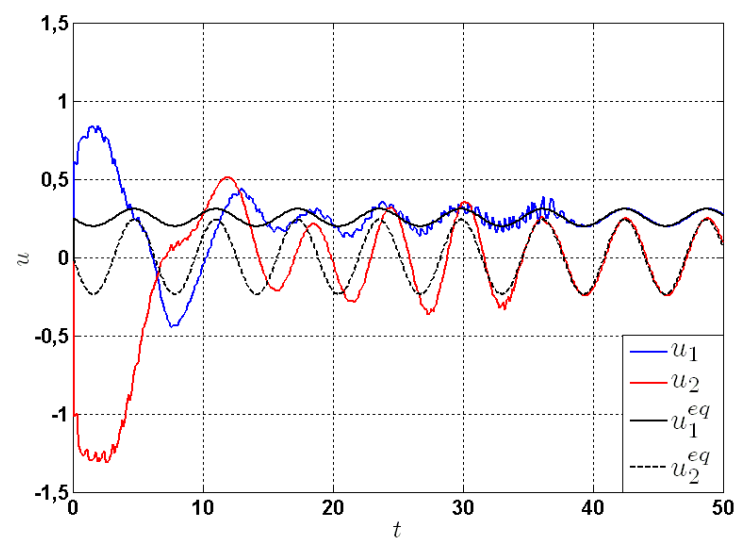

Fig. 2. Control inputs.

been selected experimentally. Remark, STEP of Algorithm 13 was realized just once on each discrete step.

The adaptive scheme of the ILF control has also been tested on this example proved almost the same control performance.

\section{CONCLUSION}

The paper presents a new approach to high order sliding mode control design, which is based on Implicit Lyapunov Function method. This approach allows us to design the control together with the Lyapunov function and to provide constructive procedures for tuning of control parameters, which are based on Linear Matrix Inequalities. Two different algorithms of practical implementation of the implicit HOSM control are presented. They achieve chattering attenuation by means of tuning of a special numerical parameter $V_{\min }$. The effectiveness of the developed numerical schemes for disturbed case was demonstrated on numerical simulations.

The ILF approach developed in this paper looks promising for many other problems such as finite-time and fixed-time observation, adaptation, formation control, etc.

\section{REFERENCES}

[1] V. Utkin, J. Guldner, and J. Shi, Sliding Mode Control in ElectroMechanical Systems. CRC Press., 2009.
[2] C. Edwards and S. Spurgeon, Sliding mode control: theory and applications. Taylor and Francis, 1998.

[3] W. Perruquetti and J. P. Barbot, Sliding Mode Control in Engineering. Marcel Dekker, 2002.

[4] A. Levant, "Homogeneity approach to high-order sliding mode design," Automatica, vol. 41, pp. 823-830, 2005.

[5] - "Principles of 2-sliding mode design," Automatica, vol. 43, pp. 576-586, 2007.

[6] - "Sliding order and sliding accuracy in sliding mode control," International Journal of Control, vol. 58, no. 6, pp. 1247-1263, 1993.

[7] A. Polyakov and A. Poznyak, "Unified Lyapunov function for a finitetime stability analysis of relay second-order sliding mode control systems," IMA Journal of Mathematical Control and Information, vol. 29(4), pp. 529-550, 2012.

[8] E. Roxin, "On finite stability in control systems," Rendiconti del Circolo Matematico di Palermo, vol. 15(3), pp. 273-283, 1966.

[9] S. Bhat and D. Bernstein, "Finite-time stability of continuous autonomous systems," SIAM Journal of Control and Optimization, vol. 38(3), pp. 751-766, 2000.

[10] E. Moulay and W. Perruquetti, "Finite time stability and stabilization of a class of continuous systems," Journal of Mathematical Analysis and Application, vol. 323, no. 2, pp. 1430-1443, 2006.

[11] Y. Orlov, "Finite time stability and robust control synthesis of uncertain switched systems," SIAM Journal of Control and Optimization, vol. 43(4), pp. 1253-1271, 2005.

[12] A. Polyakov and A. Poznyak, "Lyapunov function design for finitetime convergence analysis: "twisting" controller for second-order sliding mode realization," Automatica, vol. 45, pp. 444-448, 2009.

[13] A. Polyakov, D. Efimov, and W. Perruquetti, "Constructive finite-time and fixed-time stabilization with application to high order sliding mode control," IEEE Transactions on Automatic Control, vol. conditionally accepted, 2013

[14] Y. Hong, "Finite-time stabilization and stabilizability of a class of controllable systems," Systems \& Control Letters, vol. 46, no. 4, pp. 231-236, 2002

[15] E. Moulay and W. Perruquetti, "Finite-time stability and stabilization: State of the art," Lecture Notes in Control and Information Sciences, vol. 334, pp. 23-41, 2006.

[16] J. Moreno and M. Osorio, "Strict lyapunov functions for the supertwisting algorithm," IEEE Transactions on Automatic Control, vol. 57(4), pp. 1035-1040, 2012.

[17] A. Polyakov, "Nonlinear feedback design for fixed-time stabilization of linear control systems," IEEE Transactions on Automatic Control, vol. 57(8), pp. 2106-2110, 2012

[18] V. Korobov, "A general approach to synthesis problem," Doklady Academii Nauk SSSR, vol. 248, pp. 1051-1063, 1979.

[19] J. Adamy and A. Flemming, "Soft variable-structure controls: a survey," Automatica, vol. 40, pp. 1821-1844, 2004.

[20] A. Polyakov, D. Efimov, and W. Perruquetti, "Finite-time stabilization using implicit lyapunov function technique,", in 9th Symposium on Nonlinear Control Systems, Toulouse, France,, 4-6 September 2013, pp. $140-145$

[21] V. Zubov, "On systems of ordinary differential equations with generalized homogenous right-hand sides," Izvestia vuzov. Mathematica., vol. 1, pp. 80-88, 1958 (in Russian).

[22] H. Hermes, "Nilpotent approximations of control systems and distributions," SIAM Journal of Control and Optimization, vol. 24, pp. 731-736, 1986.

[23] L. Rosier, "Homogenous lyapunov function for homogenous continuous vector field," System \& Control Letters, vol. 19, pp. 467-473, 1992.

[24] L. Praly, "Generalized weighted homogeneity and state dependent time scale for linear controllable systems," in Proceedings of the 36th Conference on Decision \& Control, San Diego, California USA, 1997, pp. 4342-4347.

[25] H. Nakamura, N. Nakamura, and H. Nishitani, "Stabilization of homogeneous systems using implicit control lyhapunov functions," in 7th IFAC Symposium on Nonlinear Control Systems, Pretoria, Shouth Africa, 21-24 August 2007, pp. 561-566.

[26] S. Drakunov, D. Izosimov, A. Lukyanov, V. Utkin, and V. Utkin, "Block control principle i," Automation and Remote Control, vol. 51(5), pp. 601-609, 1990.

[27] A. Filippov, Differential equations with discontinuous right-hand sides. Kluwer, Dordrecht, 1988.

[28] A. Isidori, Nonlinear Control Systems. Springer, 1995. 